\title{
Supersymmetric Higgs Bosons in Weak Boson Fusion
}

\author{
Wolfgang Hollik, ${ }^{1}$ Tilman Plehn, ${ }^{2}$ Michael Rauch, ${ }^{2}$ and Heidi Rzehak ${ }^{3}$ \\ ${ }^{1}$ Max-Planck-Institut für Physik (Werner-Heisenberg-Institut), Munich, Germany \\ ${ }^{2}$ SUPA, School of Physics, University of Edinburgh, Scotland \\ ${ }^{3}$ Paul Scherrer Institut, Villigen PSI, Switzerland \\ (Received 28 April 2008; published 4 March 2009)
}

\begin{abstract}
We compute the complete supersymmetric next-to-leading-order corrections to the production of a light Higgs boson in weak-boson fusion. The size of the electroweak corrections is of similar order as the nextto-leading-order corrections in the standard model. The supersymmetric QCD corrections turn out to be significantly smaller than expected and than their electroweak counterparts. These corrections are an important ingredient to a precision analysis of the (supersymmetric) Higgs sector at the LHC, either as a known correction factor or as a contribution to the theory error.
\end{abstract}

PACS numbers: $14.80 . \mathrm{Cp}, 13.85 . \mathrm{Qk}$

The main task of the LHC era is to understand electroweak symmetry breaking and the ultraviolet completion of the standard model. According to electroweak precision data we expect to see a light Higgs boson, which should be embedded into a UV completion solving the hierarchy problem. A minimal realization of $\mathrm{TeV}$-scale supersymmetry (MSSM) is a leading candidate for that. The most promising discovery channel for a light supersymmetric Higgs boson is the production in weak-boson fusion with a subsequent decay into tau leptons [1,2]. There, a light supersymmetric Higgs scalar is guaranteed to appear over the entire MSSM parameter space [3].

Many years of LHC running will be devoted to understanding the Higgs sector in detail, for example, extracting the gauge and Yukawa couplings [4]. To meaningfully distinguish between, for example, the standard model and the MSSM-Higgs sectors [5] we need to control the theory error on the LHC rates including higher-order effects. In the standard model the next-to-leading-order QCD and electroweak corrections to weak-boson-fusion Higgs production are known to be fairly small [6]. In particular, the QCD corrections are suppressed due to the color structure of the production process and the forward-jet topology. Also interference effects between Higgs production in weak-boson fusion and in gluon fusion with two additional jets are strongly suppressed [7].

For either a comparison between the standard model and the MSSM-Higgs sectors or for a precision analysis of the MSSM Higgs sector these higher-order corrections have to be augmented by supersymmetric particle loops. In parallel to the Higgs searches, the LHC experiments will also search for direct signatures of new physics. If we should find such new states we can then predict their effects on the Higgs sector. If, for example, squarks and gluinos should be too heavy or the spectrum should be not favorable to precision MSSM analyses [8], we need to include their effects in the theory errors. Both cases require a comprehensive calculation of the supersymmetric contributions to the weakboson-fusion and gluon-fusion production processes [9].
Supersymmetry vs standard model.-Compared with its standard model counterpart the leading-order production rate of a light supersymmetric Higgs scalar $h^{0}$ includes an additional coupling factor $\sin (\beta-\alpha)$. It is expressed in terms of the ratio of the vacuum expectation values $\tan \beta$ and the scalar mixing angle $\alpha$ from the supersymmetric two-Higgs-doublet model. For a given Higgs mass we can relate the tree-level MSSM production rate to the standard model result via a simple rescaling by $\sin ^{2}(\beta-\alpha)$. For large pseudoscalar Higgs masses $m_{A} \gtrsim 200 \mathrm{GeV}$ this factor is very close to unity.

Including higher orders, there are additional contributions from the supersymmetric particle spectrum: first, we take into account loops of supersymmetric partners. If we assume $R$ parity, one-loop diagrams cannot mix supersymmetric and standard model particles, allowing for a diagram-by-diagram separation of the MSSM contributions. Second, the additional supersymmetric Higgs bosons with their standard model type $R$ charge appear in loops. As the standard model Higgs boson does not simply correspond to one supersymmetric Higgs boson we cannot separate the standard model Feynman diagrams from the MSSM set. Instead, we first compute the MSSM-Higgs corrections and then subtract the standard model Higgs loops, scaled by the tree-level correction factor.

We compute the rates using HadCalc [10] and LoopTools [11], after generating the amplitudes with FeynArts/FormCalc [12]. We assume minimal flavor violation and a $C P$-conserving MSSM. Throughout our analysis we require standard weak-boson-fusion cuts.

Higgs-sector corrections. - The mass of the light supersymmetric Higgs boson $m_{h}$ is not a free parameter. At tree level it can be computed from $m_{A}$ and $\tan \beta$. Only higherorder corrections push $m_{h}$ to values beyond the LEP2 limits [13-16]. Phenomenologically relevant studies therefore need to include quantum corrections to the Higgs mass and the Higgs potential.

The challenge in including these higher-order corrections in our calculation is that we cannot simply shift the 
final-state Higgs mass. Already in the standard model the physical Higgs mass $m_{h}$ is linked to the running quartic and top Yukawa couplings. When including self-energy corrections to the Higgs mass, we should also correct the Higgs self-couplings at the same order in perturbation theory. While this has to be done explicitly [17] in a Feynman-diagrammatic approach $[13,14]$ it is automatically taken care of if we compute the quantum effects in the scalar potential $[15,16]$.

On the other hand, quantum corrections to the Higgs potential are usually computed for vanishing external momentum instead of $m_{h}^{2}$. In the Feynman-diagrammatic approach it is straightforward to compute the Higgs selfenergies with a finite momentum flow, while additional terms have to be included for the effective potential.

Last but not least, when computing the effective scalar potential using renormalization-group techniques, it becomes increasingly tedious to separate scales, like the heavy Higgs mass $m_{A}$, the light stop mass $m_{\tilde{t}_{1}}$ and the gluino mass $m_{\tilde{g}}$.

Because the light Higgs boson is the lightest particle in the supersymmetric loops we expect the numerical effects of its mass and of the associated self-coupling $\lambda_{h h h}$ to be non-negligible. In Table I we compare the self-couplings in the effective theory [16] and in the Feynman-diagrammatic approaches [14]. Both allow for an approximate computation introducing an effective mixing angle $\alpha_{\text {eff }}$ from the scalar Higgs mass matrix. In this case the trilinear Higgs coupling $\lambda_{h h h}$ is given by the standard model coupling times the correction factor $\cos 2 \alpha_{\text {eff }} \sin \left(\beta+\alpha_{\text {eff }}\right)$. The results in this approximation should be equivalent in both schemes, because finite values of $p^{2}$ as well as corrections to the self-couplings are skipped. In Table I we see that they indeed agree very well. Between the full results, where FEYNHIGGS includes the fixed-order one-loop $\mathcal{O}\left(h_{t}^{4}\right)$ corrections to $\lambda_{h h h}$ [17], we see the expected small deviations.

Let us now turn to LHC cross sections. The supersymmetric corrections to the $V V h$ vertices $(V=W, Z)$ and the Higgs wave-function renormalization on the dominant subprocess $u d \rightarrow u d h$ we evaluate in Table II. The contributions from the Higgs sector and from supersymmetric

TABLE I. Higgs self-couplings for the parameter point SPS1a following Ref. [16] (left) and Ref. [14] (right). The common factor $-3 e m_{W} /\left(2 c_{W}^{2} s_{W}\right)$ is not included.

\begin{tabular}{|c|c|c|c|c|}
\hline & \multicolumn{2}{|c|}{ Effective theory } & \multicolumn{2}{|c|}{ Feynman diagrams } \\
\hline & $\alpha_{\text {eff }}$ & Full & $\alpha_{\text {eff }}$ & Full \\
\hline$\lambda_{H H H}$ & 0.208 & 0.198 & 0.210 & 0.210 \\
\hline$\lambda_{H H h}$ & -0.285 & -0.275 & -0.284 & -0.279 \\
\hline$\lambda_{H h h}$ & -0.216 & -0.219 & -0.220 & -0.257 \\
\hline$\lambda_{h h h}$ & 0.952 & 1.503 & 0.950 & 1.276 \\
\hline$\alpha_{\text {eff }}$ & \multicolumn{2}{|c|}{-0.1132} & \multicolumn{2}{|c|}{-0.1158} \\
\hline$m_{h}$ & \multicolumn{2}{|c|}{$109.8 \mathrm{GeV}$} & \multicolumn{2}{|c|}{$111.0 \mathrm{GeV}$} \\
\hline$m_{H}$ & \multicolumn{2}{|c|}{$391.5 \mathrm{GeV}$} & \multicolumn{2}{|c|}{$391.6 \mathrm{GeV}$} \\
\hline
\end{tabular}

particles cannot be separated because of their combined renormalization. The upper lines use a wave-function renormalization at one loop. In the $\alpha_{\text {eff }}$ approximation they agree well, despite the different external Higgs masses. At the bottom we include a higher-order improved wavefunction renormalization [14]. In this Letter we will use the Feynman-diagrammatic approach with one-loop $Z$ factors, to allow for a proper renormalization-scale behavior. The difference of $0.05 \%$ between the different rate predictions is a lower limit on the theory error from the MSSM Higgs sector. Note, however, that this theory error only applies if we strictly assume the minimal renormalizable supersymmetric Higgs sector.

Supersymmetric particle corrections.-Example oneloop diagrams appearing in the $q q \rightarrow q q h$ processes we show in Fig. 1. In Ref. [9] only the SUSY-QCD vertex corrections, the left diagram of Fig. 1, have been computed so far and we find agreement. Our numerical results are based around the parameter point SPS (Snowmass points and slopes) 1a [18], for which there are two aspects we need to remember: supersymmetric particles with only electroweak charges have typical masses around $200 \mathrm{GeV}$, while squarks and gluinos range around $600 \mathrm{GeV}$, and $\tan \beta=10$ avoids large nondecoupling effects from down-type fermions.

As we can see in Table III all QCD corrections $\Delta \sigma \propto$ $\alpha^{3} \alpha_{s}$ turn out to be surprisingly small. In the standard model we know that from a QCD perspective we are essentially looking at two-sided noninterfering deep inelastic scattering. However, we find an even larger suppression in the supersymmetric case.

Of the vertices only the $q q^{\prime} W$ and $q q Z$ ones are corrected by squark or gluino loops. Since the $\tilde{q} \tilde{q}^{\prime} W$ coupling connects left-handed sfermions and the left-right mixing of light-flavor squarks is negligible, both external quarks are left handed, just as at tree level. This means that in the oneloop diagram (closed with the Born diagram) the fermion trace cannot include a gluino-mass insertion, which would require a chirality flip. Instead, the typical momentum scale in the numerator is an order of magnitude below the gluino mass in the denominator. In the electroweak case, also the lighter charginos and neutralinos in the loop

TABLE II. Schemes for the supersymmetric corrections to the $V V h$ vertices for the leading subprocess at the hadronic level.

\begin{tabular}{|c|c|c|}
\hline & $\Delta \sigma / \sigma(u d \rightarrow u d h)$ & $\left(\sigma_{\alpha_{\text {eff }}}-\sigma_{\text {full }}\right) / \sigma$ \\
\hline & \multicolumn{2}{|c|}{ Effective theory } \\
\hline$\alpha_{\text {eff }}$ & $-0.389 \%$ & \multirow{2}{*}{$-0.122 \%$} \\
\hline \multirow[t]{2}{*}{ Full } & $-0.266 \%$ & \\
\hline & \multicolumn{2}{|c|}{ Feynman diagrams } \\
\hline$\alpha_{\text {eff }}$ & $-0.393 \%$ & $-0076 \%$ \\
\hline \multirow[t]{2}{*}{ Full } & $-0.317 \%$ & $-0.0 / 6 \%$ \\
\hline & Feynman diagr & mproved $Z_{\mathrm{FH}}$ \\
\hline$\alpha_{\text {eff }}$ & $-0.343 \%$ & $-0115 \%$ \\
\hline Full & $-0.228 \%$ & $-0.115 \%$ \\
\hline
\end{tabular}



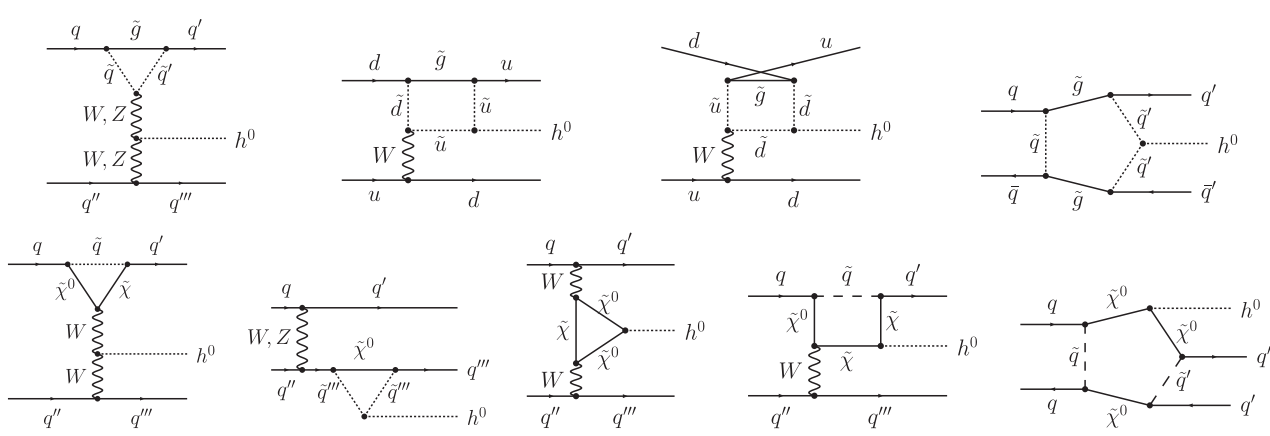

FIG. 1. Feynman diagrams contributing to QCD (upper) and electroweak (lower) vertex corrections, boxes, and pentagons.

couple to the vector boson. This means we can add a double mass insertion into the fermion line which can partly compensate for the heavy masses in the loop denominator. This effect and the corrections to the $V V h$ vertex lead to a relative enhancement of the electroweak over the QCD $q q V$ vertex correction in Table III.

In both box diagrams shown in Fig. 1 the $\tilde{q} \tilde{q}^{\prime} W$ and $q \tilde{q} \tilde{g}$ couplings are the same, but the $\tilde{q} \tilde{q} h$ coupling is proportional to $T_{3}-Q s_{w}^{2}$, i.e., around $-1 / 3$ for down squarks and $+5 / 16$ for up squarks. This leads to a cancellation by 1 order of magnitude, which cannot be broken by different squark masses either, because left-handed squarks form an $S U$ (2) doublet. This argument does not hold for the subleading $\mathrm{ZZ}$ fusion, where we indeed find that the corrections turn out to be at a more natural level.

The exchange of a gluon or gluino between the two incoming quarks is proportional to the trace of the $S U(3)$ generators and hence zero. In Fig. 1, we show another supersymmetric pentagon diagram with two colored particles exchanged, which gets rid of the color suppression. Such diagrams contribute formally at order $\mathcal{O}\left(\alpha_{s}^{2} \alpha^{2}\right)$, which is as large as the Born term $\mathcal{O}\left(\alpha^{3}\right)$. However, their kinematic properties are completely different from the vector-boson-fusion topology and the large loop masses further reduce their contribution to a negligible level.

Following the above arguments, the supersymmetric QCD corrections to weak-boson-fusion Higgs production are suppressed by a whole list of mechanisms, which

TABLE III. Complete supersymmetric corrections to the process $p p \rightarrow q q h$ by diagrams. Our parameter point SPS1a has a tree-level rate of $706 \mathrm{fb}$.

\begin{tabular}{lccc}
\hline \hline Diagram & $\Delta \sigma / \sigma[\%]$ & Diagram & $\Delta \sigma / \sigma[\%]$ \\
\hline$\Delta \sigma \sim \mathcal{O}(\alpha)$ & & \multicolumn{2}{c}{$\Delta \sigma \sim \mathcal{O}\left(\alpha_{s}\right)$} \\
Self-energies & 0.199 & & \\
$q q^{\prime} W+q q Z$ & -0.392 & $q q^{\prime} W+q q Z$ & -0.0148 \\
$q q h$ & -0.0260 & $q q h$ & 0.00545 \\
$W W h+Z Z h$ & -0.329 & & \\
Box & 0.0785 & Box & -0.00518 \\
Pentagon & 0.000522 & Pentagon & -0.000308 \\
Sum of all $\Delta \sigma / \sigma=-0.484 \%$ & & \\
\hline \hline
\end{tabular}

explain their at first sight surprising suppression even with respect to the electroweak corrections in Table III.

More generally, we show the next-to-leading-order corrections for all SPS parameter points [18] in Table IV. From the discussion above we do not expect the picture of electroweak vs strong corrections to change significantly. Heavier supersymmetric spectra and different values of $\tan \beta$ and of the trilinear couplings just scale the overall size of the supersymmetric corrections. The relatively large corrections for SPS5 are driven by a light top squark, while the largely decoupled spectrum in SPS9 leads to negligible MSSM effects. The typical size of the complete MSSM corrections is less or around $1 \%$.

To study the behavior of the one-loop corrections with varying supersymmetric masses we use the parameter point SPS1b with a universal gaugino mass between 100 and $1000 \mathrm{GeV}$. In Fig. 2 we show the result for a $m_{1 / 2}$-dependent Higgsino mass parameter as well as for the fixed SPS1b value $\mu=499 \mathrm{GeV}$. The corrections sharply drop with increasing $m_{1 / 2}$ as we approach the decoupling limit. Fixing $\mu$ means larger corrections for a light SUSY spectrum and a sharper drop for heavy masses. The maximum size for the corrections consistent with the LEP2 chargino limit we read off to be $-2 \%$. If we tune all weak-scale MSSM parameters to barely respect all LEP2 and Tevatron limits we find that the size of the supersym-

TABLE IV. Complete MSSM corrections for all SPS parameter points [18]. The vertex correction in the first column corresponds to Table II, but including all partonic channels.

\begin{tabular}{lcccc}
\hline \hline & \multicolumn{4}{c}{$\Delta \sigma / \sigma[\%]$} \\
& $W W h+Z Z h$ & $\mathcal{O}(\alpha)$ & $\mathcal{O}\left(\alpha_{s}\right)$ & all \\
\hline SPS1a & -0.329 & -0.469 & -0.015 & -0.484 \\
SPS1b & -0.162 & -0.229 & -0.006 & -0.235 \\
SPS2 & -0.147 & 0.129 & -0.002 & -0.131 \\
SPS3 & -0.146 & -0.216 & -0.006 & -0.222 \\
SPS4 & -0.258 & -0.355 & -0.008 & -0.363 \\
SPS5 & -0.606 & -0.912 & -0.010 & -0.922 \\
SPS6 & -0.226 & -0.309 & -0.010 & -0.319 \\
SPS7 & -0.206 & -0.317 & -0.006 & -0.323 \\
SPS8 & -0.157 & -0.206 & -0.004 & -0.210 \\
SPS9 & -0.094 & -0.071 & -0.003 & -0.074 \\
\hline \hline
\end{tabular}




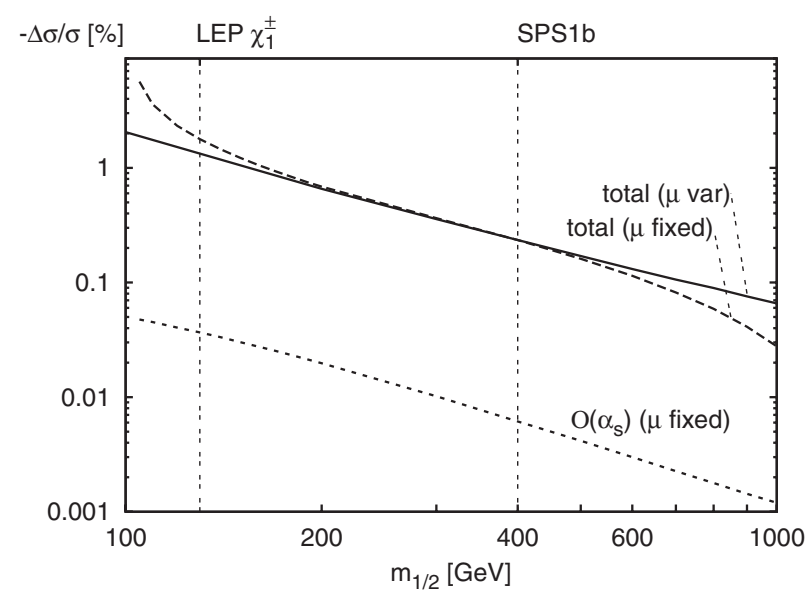

FIG. 2. Next-to-leading-order corrections as a function of $m_{1 / 2}$ for varying and for fixed $\mu$. For the latter we also show the strong corrections. The vertical lines indicate the LEP2 chargino limit and the reference point SPS1b.

metric corrections is bounded by $-4 \%$. Explicit nondecoupling effects in the bottom Yukawa only appear at the twoloop level, so all curves in Fig. 2 decouple smoothly for increasing masses. Consistent with our previous discussion the $\mathcal{O}\left(\alpha_{s}\right)$ corrections are negligible over the entire parameter range.

Outlook. - In the light of a possible precision analysis of the standard model and MSSM Higgs sectors at the LHC we have analyzed the supersymmetric one-loop corrections to the weak-boson-fusion production process.

The appearance of all supersymmetric neutral Higgs bosons in the loops required us to study different methods of describing higher-order effects on Higgs masses and the Higgs potential. We find corrections from the Higgs sector at the percent level, with a remaining uncertainty of below $0.1 \%$ reflecting unknown higher-order corrections.

The complete set of electroweak loop diagrams contributes at the percent level, as is expected for massive $\mathcal{O}(\alpha)$ corrections. Compared with those, the (known) supersymmetric one-loop QCD corrections are negligible. This is due to a variety of effects, based on the color structure, the supersymmetric coupling structure, or the kinematics of the process.

In total, the supersymmetric one-loop corrections to Higgs production via vector-boson fusion can amount to $4 \%$ for parameter points allowed by SUSY searches and are typically at or below $1 \%$. Their sign is negative. This result should serve as a solid basis for a precision analysis of the supersymmetric Higgs sector at the LHC.

We would like to thank Dominik Stöckinger and Thomas Binoth for valuable discussions. We would also like to thank the PhenoGrid VO and ScotGrid for providing computer resources. This work was supported in part by the European Community's Marie-Curie Research Training Network under Contract No. MRTN-CT-2006035505 (HEPTOOLS).
[1] S. Asai et al., Eur. Phys. J. C 32S2, s19 (2004).

[2] D. L. Rainwater, D. Zeppenfeld, and K. Hagiwara, Phys. Rev. D 59, 014037 (1998); T. Plehn, D. L. Rainwater, and D. Zeppenfeld, Phys. Rev. D 61, 093005 (2000).

[3] T. Plehn, D. L. Rainwater, and D. Zeppenfeld, Phys. Lett. B 454, 297 (1999).

[4] M. Dührssen et al., Phys. Rev. D 70, 113009 (2004).

[5] A. Djouadi, Phys. Rep. 457, 1 (2008); 459, 1 (2008).

[6] T. Han, G. Valencia, and S. Willenbrock, Phys. Rev. Lett. 69, 3274 (1992); M. Spira, Fortschr. Phys. 46, 203 (1998); T. Figy, D. Zeppenfeld, and C. Oleari, Phys. Rev. D 68, 073005 (2003); M. Ciccolini, A. Denner, and S. Dittmaier, Phys. Rev. Lett. 99, 161803 (2007); Phys. Rev. D 77, 013002 (2008).

[7] J. R. Andersen, T. Binoth, G. Heinrich, and J. M. Smillie, J. High Energy Phys. 02 (2008) 057; A. Bredenstein, K. Hagiwara, and B. Jäger, Phys. Rev. D 77, 073004 (2008).

[8] P. Bechtle, K. Desch, W. Porod, and P. Wienemann, Eur. Phys. J. C 46, 533 (2006); R. Lafaye, T. Plehn, M. Rauch, and D. Zerwas, Eur. Phys. J. C 54, 617 (2008).

[9] A. Djouadi and M. Spira, Phys. Rev. D 62, 014004 (2000); M. Mühlleitner and M. Spira, Nucl. Phys. B790, 1 (2008); C. Anastasiou, S. Beerli, and A. Daleo, Phys. Rev. Lett. 100, 241806 (2008).

[10] M. Rauch, arXiv:0804.2428.

[11] T. Hahn and M. Rauch, Nucl. Phys. B, Proc. Suppl. 157, 236 (2006); A. Denner and S. Dittmaier, Nucl. Phys. B658, 175 (2003).

[12] T. Hahn, Comput. Phys. Commun. 140, 418 (2001); T. Hahn and C. Schappacher, Comput. Phys. Commun. 143, 54 (2002); T. Hahn and M. Perez-Victoria, Comput. Phys. Commun. 118, 153 (1999).

[13] P. H. Chankowski, S. Pokorski, and J. Rosiek, Nucl. Phys. B423, 437 (1994); R. Hempfling and A. H. Hoang, Phys. Lett. B 331, 99 (1994); D. M. Pierce, J. A. Bagger, K. T. Matchev, and R. J. Zhang, Nucl. Phys. B491, 3 (1997); S. Heinemeyer, W. Hollik, and G. Weiglein, Phys. Rev. D 58, 091701(R) (1998); R. J. Zhang, Phys. Lett. B 447, 89 (1999); J. R. Espinosa and R. J. Zhang, J. High Energy Phys. 03 (2000) 026; Nucl. Phys. B586, 3 (2000).

[14] M. Frank and H. Rzehak et al., J. High Energy Phys. 02 (2007) 047.

[15] H.E. Haber and R. Hempfling, Phys. Rev. D 48, 4280 (1993); J. A. Casas, J. R. Espinosa, M. Quiros, and A. Riotto, Nucl. Phys. B436, 3 (1995); B439, 466(E) (1995); M.S. Carena, M. Quiros, and C.E.M. Wagner, Nucl. Phys. B461, 407 (1996); H. E. Haber, R. Hempfling, and A. H. Hoang, Z. Phys. C 75, 539 (1997); S. P. Martin, Phys. Rev. D 66, 096001 (2002); 71, 016012 (2005).

[16] M. S. Carena, J. R. Espinosa, M. Quiros, and C.E. M. Wagner, Phys. Lett. B 355, 209 (1995).

[17] W. Hollik and S. Penaranda, Eur. Phys. J. C 23, 163 (2002); A. Dobado, M. J. Herrero, W. Hollik, and S. Penaranda, Phys. Rev. D 66, 095016 (2002); see also J. Brod, F. Fugel, and B. A. Kniehl, Phys. Rev. D 78, 011303(R) (2008).

[18] B. C. Allanach et al., Eur. Phys. J. C 25, 113 (2002). 